\title{
Limitations of middle cerebral artery peak systolic velocity in the detection of severe anaemia: A case report
}

\author{
L Geerts, ${ }^{1}$ MB ChB, MRCOG, BSc Hons (Human Genetics); J N Rossouw, ${ }^{1}$ MB ChB, MMed (O\&G), FCOG (SA); \\ A C van Wyk, ${ }^{2,3}$ MMed (Anat Path); C A Wright, ${ }^{2,4}$ FRCPath, PhD

\begin{abstract}
${ }^{1}$ Department of Obstetrics and Gynaecology, Tygerberg Hospital and Faculty of Medicine and Health Sciences, Stellenbosch University, Tygerberg, Cape Town, South Africa

${ }^{2}$ Division of Anatomical Pathology, Faculty of Medicine and Health Sciences, Stellenbosch University, Tygerberg, Cape Town, South Africa

${ }^{3}$ National Health Laboratory Service, Tygerberg Hospital, Cape Town, South Africa

${ }^{4}$ National Health Laboratory Service, Port Elizabeth, South Africa
\end{abstract}

Corresponding author: L Geerts (lgeerts@sun.ac.za)

\begin{abstract}
Doppler examination of the fetal middle cerebral artery (MCA) is considered highly reliable in detecting severe fetal anaemia. We present a case of a mother with severe pre-eclampsia at 32 weeks' gestation and non-immune fetal hydrops without obvious cause. Since the MCA peak systolic velocity (PSV) was normal, severe anaemia was not considered as a possible cause, but after delivery the infant was found to be severely anaemic and died. Histological examination of the placenta revealed multiple extensive haemorrhages (subchorionic, intraparenchymal and intravillous), and autopsy indicated that the most likely cause of death was severe anaemia and hypoxia due to multiple and extensive placental haemorrhages, which had developed over the course of several days. We postulate that the MCA PSV failed to indicate severe anaemia because of the combination of subacute anaemia with severe profound hypoxia due to placental insufficiency.
\end{abstract}

S Afr J Obstet Gynaecol 2015;21(2):44-45. DOI:10.7196.SAJOG.898

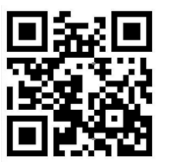

Doppler assessment of the peak systolic flow velocity (PSV) of the middle cerebral artery (MCA) is highly reproducible in trained hands and very effective in detecting severe anaemia of all causes. ${ }^{[1]}$ In cases of fetal hydrops it guides management in terms of fetal treatment, expectant care or urgent delivery. We present a case of nonimmune hydrops fetalis (NIHF) where the MCA PSV was normal despite postnatal evidence of severe anaemia.

\section{Case report}

A 19-year-old healthy Rh-positive primigravida initiated antenatal care at 9 weeks with negative tests for syphilis and HIV. She had a normal ultrasound scan at 20 weeks and five uncomplicated visits before presenting at 28 weeks with generalised oedema, decreased fetal movements and pre-eclampsia. Ultrasound examination revealed a single male fetus with anasarca, bilateral hydrothorax, a pericardial effusion, cardiomegaly, severe ascites, polyhydramnios and decreased fetal movements. There was absent end-diastolic flow in the umbilical artery, a high pulsatility index (PI) (1.12) in the ductus venosus (positive a-wave) and a low MCA-PI (1.21). In view of the normal MCA PSV $(40 \mathrm{~cm} / \mathrm{s})$, anaemia was considered unlikely. In view of the early gestation, severity of the hydrops, absence of an overt treatable cause and lack of an intensive care bed at the time, the mother agreed to defer active intervention while awaiting special investigations (infectious work-up negative), betamethasone administration and maternal stabilisation. Several days later, she experienced a small antepartum haemorrhage with irregular contractions, and after an unmonitored labour a $1820 \mathrm{~g}$, very pale and oedematous male infant was delivered vaginally with Apgar scores of 1/1/1. Extensive resuscitation was withheld, and the infant died. Skin biopsy by quantitative fluorescent polymerase chain reaction was normal (for chromosomes 13, 18, 21, X and Y).

Autopsy revealed no additional macroscopic abnormalities. Extensive extramedullary haematopoiesis was seen in the liver, and fetal vessels contained moderately increased nucleated red blood cells (NRBCs),

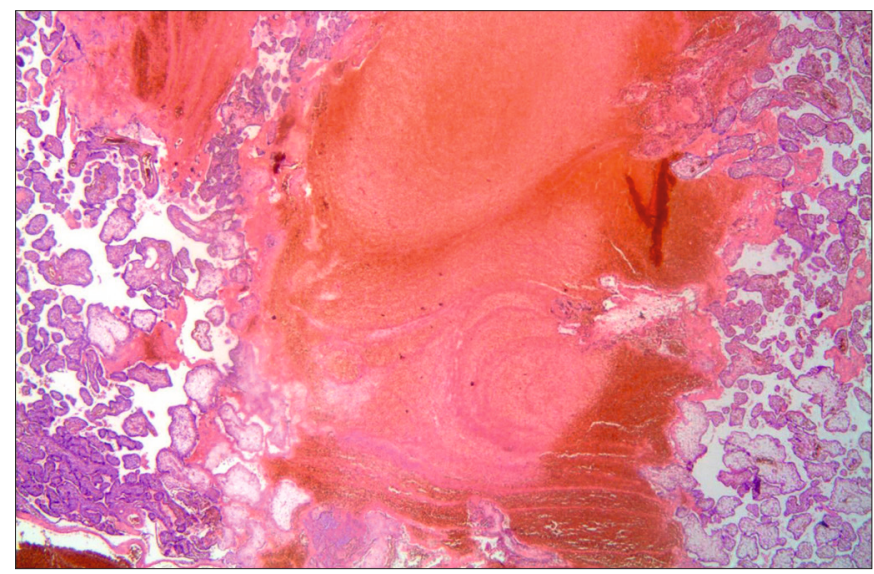

Fig. 1. Laminated intraparenchymal placental thrombus (haematoxylin and eosin stain, original magnification $\times 20$ ).

without intranuclear inclusions typical for parvovirus infection. Neuropathology showed a grade 2 germinal matrix and subarachnoidal haemorrhage, both small and regarded as manifestations of terminal hypoxia rather than the cause of death. Starry-sky appearance of the thymus with loss of corticomedullary differentiation and pseudoacinar formation in the adrenal cortex suggested a period of intrauterine stress of at least a few days, consistent with hypoxia. The placenta was enlarged (460 g v. expected 229 - $357 \mathrm{~g}$ ) and showed a 15\% retroplacental haemorrhage, one large $(45 \mathrm{~mm})$ subchorionic and multiple small intraparenchymal haemorrhages with delayed villous maturation, marked diffuse villous oedema, multifocal intravillous haemorrhages containing NRBCs, and laminated intraparenchymal thrombi (Fig. 1).

\section{Discussion}

This report describes a case of fetomaternal hydrops due to multiple and extensive placental haemorrhages and questions why the MCA PSV did 
not suggest severe anaemia as the underlying cause. This is of particular concern in view of the fact that fetal anaemia is one of the treatable causes of NIHF and associated with the best prognosis if actively managed, either by urgent delivery or intrauterine transfusion. We postulate that the MCA PSV was not raised because of the combination of hypoxia due to placental insufficiency and possibly episodes of hypovolaemia, superimposed on the effects of (sub)acute anaemia due to fetal blood loss.

Fetal hydrops has many causes and carries a very high mortality rate. Approximately $10 \%$ of cases are caused by haematological disorders, with cardiac failure resulting from loss of oxygen-carrying capacity from either reduced or abnormal production or excessive loss of erythrocytes. Large fetomaternal haemorrhages (FMHs) are rare ( 1\%) and can cause perinatal death and severe acute and long-term morbidity, but most occur unexpectedly before labour, and they are often chronic or intermittent. ${ }^{[2]}$ Classic presenting symptoms include reduced fetal movements. Typical postmortem findings include severe pallor, hydrops, collapsed heart and vessels and histopathological features of chronic stress. ${ }^{[3]}$ Typical placental findings are retroplacental haemorrhages and (laminated) intervillous thrombi with NRBCs in fetal organs and intervillous spaces. ${ }^{[4]}$ Laminated intervillous thrombi (linear streaks of fibrin with degenerating red and white blood cells in between) are thought to be caused by leakage of fetal blood into the intervillous spaces via defects in the trophoblast, usually occurring at varying times and leading to clotting of mixed fetal-maternal blood as a mechanism to limit fetal blood loss and fetomaternal bleeding. ${ }^{[4]}$

The utility of MCA PSV screening for fetal anaemia is based on the compensatory circulatory responses (aimed at maintaining tissue oxygenation) seen with non-acute progressive anaemia in fetuses with normal cardiovascular physiology and normal placental respiratory function. Initially decreased viscosity after haemodilution improves venous return and cardiac preload, thereby increasing cardiac output. With worsening anaemia, the resulting hypoxaemia initially causes redistribution of blood flow to the brain and coronary arteries, but ultimately tissue hypoxia leads to metabolic acidosis and myocardial dysfunction, manifesting as right atrial enlargement and placentomegaly due to congestion, and later overt fetal hydrops and demise due to high-output cardiac failure.

The hyperdynamic fetal circulation with subacute or moderate chronic anaemia has been mainly researched (and is now routinely used) in pregnancies with red cell isoimmunisation. In well-grown fetuses, the MCA PSV increase is related to the haemoglobin but not the partial pressure of oxygen $\left(\mathrm{pO}_{2}\right)$, suggesting that this reflects decreased blood viscosity and increased venous return rather than an active fetal compensatory mechanism by $\mathrm{pO}_{2}$-related chemoreceptor stimulation (to increase cardiac contractility and cause vasodilatation). ${ }^{[1,5,6]}$ Reports on MCA PSV in anaemia from other causes (parvovirus, haemoglobinopathies, inter-twin transfusion, haemorrhage, etc.) and in hydropic fetuses are limited, but a few authors have found a similarly high sensitivity of MCA velocity for severe anaemia in fetal hydrops, both immune and non-immune. ${ }^{[1,7]}$ With (sub)acute severe fetal blood loss, however, the fetus may not adapt in a similar way, and while some cases of increased MCA PSV with acute fetal haemorrhage have been reported, no studies have systematically assessed the diagnostic accuracy of MCA PSV in those circumstances. Among 15 women with FMH, Cosmi et al. ${ }^{[7]}$ found MCA PSV of value in 9 women with chronic abruption, but in 5 with acute abruption the result was false-negative.

Changes in MCA blood flow are also seen with fetal growth restriction (FGR) with placental insufficiency. The associated chronic fetal hypoxaemia results in redistribution of blood flow in favour of vital organs including the brain, initially characterised by decreasing MCA impedance due to increasing vasodilatation with worsening hypoxaemia. ${ }^{[8]}$ MCA velocity changes in FGR have been less extensively studied, but Vyas et al. ${ }^{[8]}$ showed increasing MCA mean velocity with worsening fetal hypoxaemia and acidaemia in 81 FGR fetuses, possibly related to decreasing cerebral and increasing placental vascular resistance with redistribution of cardiac output (in favour of the left ventricle). Although FGR fetuses initially increase their red blood cell mass to improve oxygenation, they often become progressively anaemic as a result of nutritional problems, insufficient bone marrow response, microvascular consumption or dyserythropoiesis. Since the degree of anaemia could be an indicator of the severity of placental insufficiency, MCA PSV screening in FGR fetuses has been of interest. In contrast to alloimmunised fetuses, Hanif et al. ${ }^{[6]}$ found no correlation between MCA PSV and fetal haemoglobin in FGR but confirmed a correlation between MCA PSV and partial carbon dioxide pressure $\left(\mathrm{pCO}_{2}\right)$ and $\mathrm{pO}_{2}$. Makh et al. ${ }^{[9]}$ found MCA PSV sensitivity for severe anaemia unacceptably low in 97 FGR fetuses; this was possibly explained by the attenuating effect of the decreased cerebral vascular resistance on the PSV elevation in combination with reduced forward cardiac function due to the increased afterload and intrinsic hypoxia-related cardiac dysfunction. MCA PSV is therefore clearly affected by different, competing mechanisms. The coexistence of significant hypoxaemia and anaemia may affect blood flow patterns in an unpredictable way as cardiovascular dynamics change in FGR and oxygen delivery to the tissues is further decreased.

This patient presented with reduced fetal movements of several days' duration and the ultrasound features were typical of severe anaemia (fetomaternal hydrops with dominant ascites). Examination of the infant and placenta concurred with a relatively protracted course (severe pallor, changes in the adrenal gland and thymus, intervillous laminated thrombi, subchorionic, retroplacental and intraparenchymal haemorrhages). The MCA PSV would have been expected to reliably exclude severe anaemia in this non-acute setting. However, the placental pathology was of a nature that would have caused both severe chronic fetal hypoxaemia and (chronic and/ or subacute) anaemia, and the Doppler results indeed indicated very poor placental perfusion with significant redistribution and cardiac compromise. The degree of fetal hypoxaemia in this case would have been disproportionate to the degree of anaemia seen in alloimmunised pregnancies, and the severe anaemia (resulting both from placental deprivation and recurrent blood loss) would have compromised the chronic poor fetal tissue oxygenation even further. This combination probably contributed to significant impairment of forward cardiac function, which may have prevented the fetus from increasing the MCA PSV in response to severe anaemia.

\section{Conclusion}

Clinicians should be aware of the limitation of MCA PSV screening for anaemia in cases where severe placental insufficiency is coexistent, since a potentially treatable cause of hydrops may be missed and a more active approach to management (either in utero intervention or urgent delivery) could be appropriate.

1. Schenone MH, Mari G. The MCA Doppler and its role in the evaluation of fetal anemia and fetal growth restriction. Clin Perinatol 2011;38(1):83-102. [http://dx.doi.org/10.1016/j.clp.2010.12.003] 2. Giacoia GP. Severe fetomaternal haemorrhage: A review. Obstet Gynecol Surv 1997;52(6):372-380

3. Biankin SA, Arbuckle SM, Graf NS. Autopsy findings in a series of five cases of fetomaternal haemorrhages. Pathology 2003;35(4):319-324.

4. Devi B, Jennison RF, Langley FA. Significance of placental pathology in transplacental haemorrhage. J Clin Pathol 1968;21(3):322-331.

5. Picklesimer AH, Oepkes D, Moise KJ Jr, et al. Determinants of the middle cerebral artery peak systolic velocity in the human fetus. Am J Obstet Gynecol 2007;197(5):526.el-e4. [http://dx.doi. org/10.1016/j.ajog.2007.04.002]

6. Hanif F, Drennan K, Mari G. Variables that affect the middle cerebral artery peak systolic velocity

in fetuses with anemia and intrauterine growth restriction. Am J Perinatol 2007;24(8):501-505. in fetuses with anemia and intrauterine g
[http://dx.doi.org/10.1055/s-2007-986683]

7. Cosmi E, Rampon M, Saccardi C, Zanardo V, Litta P. Middle cerebral artery peak systolic velocity in the diagnosis of fetomaternal hemorrhage. Int J Gynaecol Obstet 2012;117(2):128-130. [http:// dx.doi.org/10.1016/j.ijgo.2011.12.016]

8. Vyas S, Nicolaides KH, Bower S, Campbell S. Middle cerebral artery flow velocity waveforms in fetal hypoxaemia. Br J Obstet Gynaecol 1990;97(9):797-803.

9. Makh DS, Harman CR, Baschat AA. Is Doppler prediction of anemia effective in the growth restricted fetus? Ultrasound Obstet Gynecol 2003;22(5):489-492. [http://dx.doi.org/10.1002/uog.206] 